\title{
Remarkable Diagnostic Magnetic Resonance Imaging Findings in Sellar Xanthogranuloma: Report of Three First Cases in Latin America
}

\author{
Mario Tapia Céspedes ${ }^{1}$ Jaime Pinto Vargas ${ }^{2}$ Fernando Andrade Yañez ${ }^{3}$ Loreto Spencer León ${ }^{4}$ \\ Pablo Álvarez Arancibia ${ }^{5}$ Thomas Schmidt Putz ${ }^{6}$ \\ ${ }^{1}$ Department of Otorhinolaryngology - Head and Neck Surgery, \\ Regional Hospital of Concepción, Concepción, Chile \\ ${ }^{2}$ Department of Neurosurgery, Regional Hospital of Concepción, \\ Concepción, Chile \\ ${ }^{3}$ Department of Radiology, Regional Hospital of Concepción, \\ Concepción, Chile \\ ${ }^{4}$ Department of Pathology, Regional Hospital of Concepción, \\ Concepción, Chile \\ ${ }^{5}$ Faculty of Medicine, University of Concepción, Concepción, Chile \\ ${ }^{6}$ Department of Otorhinolaryngology - Head and Neck Surgery, \\ University of Concepción, Chile \\ J Neurol Surg Rep 2017;78:e26-e33. \\ Address for correspondence Thomas W. Schmidt Putz, MD, \\ Department of Otorhinolaryngology - Head and Neck Surgery, \\ Specialty Department, University of Concepción, Chacabuco Avenue, \\ No.1401, Concepción, Chile (e-mail: Schmidtp@udec.cl).
}

\begin{abstract}
Keywords

- xanthogranuloma

- craniopharyngioma

- sellar region

- MRI
\end{abstract}

\section{Introduction}

The World Health Organization (WHO) appointed xanthogranuloma of the sellar region as a specific type of brain tumor in 2000, differentiating it from adamantinomatous craniopharyngioma (CP). They are a rare entity, with two cases reported in the Western Hemisphere. ${ }^{1,2}$ Histologically, xanthogranulomas correspond to a granulomatous reaction characterized by the presence of cholesterol crystals, foamy macrophages, giant cells, hemosiderin deposits, necrotic detritus, lymphocytic infiltrate, and fibrous proliferation. ${ }^{3}$ Since the 37 cases were reported by Paulus et al, ${ }^{3} 21$ reports have been documented since 2000 , without previous reports in Latin America. ${ }^{2,4-23}$

The etiology of xanthogranulomas of the sellar region is controversial, and there are currently two hypotheses under greater discussion. The first states that xanthogranulomas originate from an inflammatory reaction, hemorrhage or rupture of a Rathke's cleft cyst (RCC). The second hypothesis postulates that xanthogranulomas arise from a secondary inflammatory progression of a CP. ${ }^{19,21,24}$ Until now, there is no preoperative diagnostic method, imaging and immunohistochemical studies have not been sufficient by themselves to achieve accurate preoperative diagnosis. ${ }^{1,9,20,21}$ We report three cases of sellar xanthogranuloma, highlighting an imaging finding in one of the cases that might be helpful to elucidate a characteristic pattern of these lesions.

\section{Case 1}

A 10-year-old patient, obese, with no other relevant medical history, presented with a 1-year history of recurrent received

July 17, 2016

accepted after revision

December 21, 2016
DOI http://dx.doi.org/ 10.1055/s-0037-1598203. ISSN 2193-6358. (c) 2017 Georg Thieme Verlag KG Stuttgart · New York
License terms

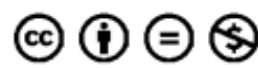



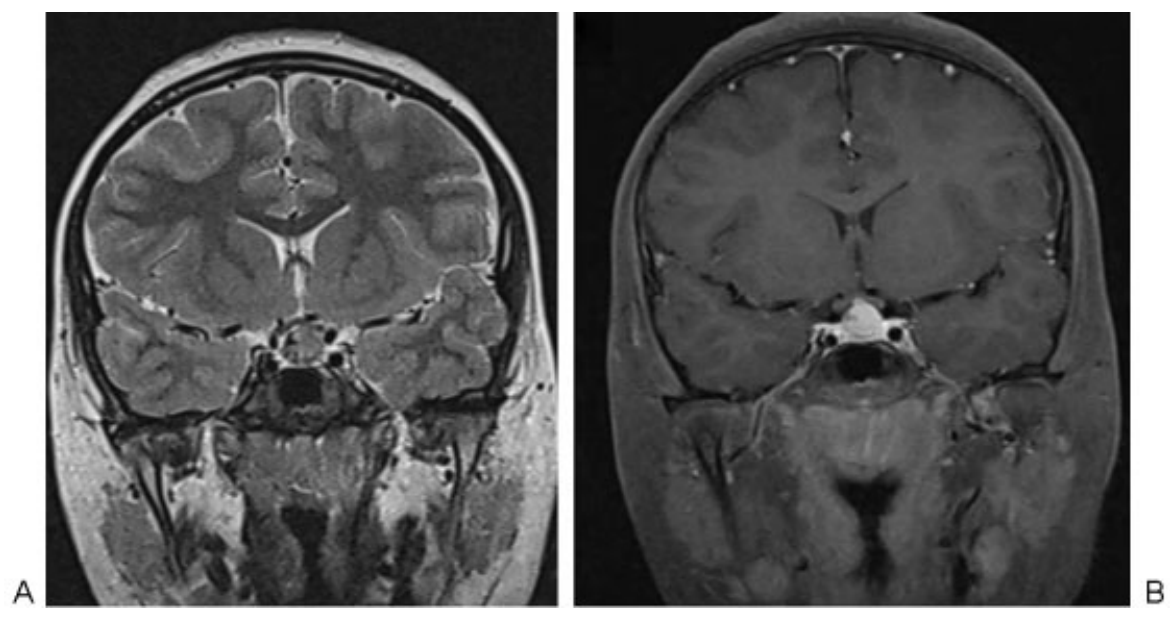

Fig. 1 Case 1. (A) Coronal T2 and (B) coronal T1 postcontrast. Intrasuprasellar cystic hyperintense tumor with small solid hypointense peripheric nodule.

episodes of headache associated with blurred vision, polyuria, and polydipsia. Magnetic resonance imaging (MRI) of the brain showed a cystic solid heterogeneous sellar mass with suprasellar extension, suggestive of $\mathrm{CP}$ or pituitary adenoma (-Figs. 1 and 2 ). Goldmann perimetry showed a tubular visual field unrelated to the pituitary lesion.

An extended endoscopic transsphenoidal, transplanum, transselar, transtuberculum approach was performed. Macroscopically, the mass was described as similar to a CP with an old hematoma at its center. The patient had no postoperative complications. Histological examination revealed findings consistent with xanthogranuloma. At 38 months' follow-up after surgery, the patient had a significant improvement in visual disturbances, with no more headache episodes, polyuria, and polydipsia. The postoperative MRI confirmed the total tumor resection without recurrence or residual disease.

\section{Case 2}

A 35-year-old male patient, with a history of dyslipidemia, presented a 2-year history of recurrent headache, progressive visual loss, blurred vision, decreased libido and erectile dysfunction. The visual field test showed a superior temporal quadrantanopsia in the left eye and a temporal hemianopsia in the right eye. Endocrinological evaluation showed hypogonadism associated with secondary adrenal insufficiency. The MRI showed an expansive process of the sellarsuprasellar region, partially cystic, slightly heterogeneous, hyperintense on T1-weighted and heterogeneous in T2-weighted image with calcifications, mass effect on the optic chiasm, and a retroclival dural tail with contrast enhancement (-Fig. 3).

An extended endoscopic transsphenoidal, transselar, transtuberculum, transplanum approach was made without complications. Histopathological examination showed the presence of cholesterol crystals, sclerosis and fibrosis of the connective tissue, cholesterol granulomas, lymphocytic infiltrate, hemosiderin deposits, and multinucleated giant cells without epithelial component, findings consistent with a xanthogranuloma (-Fig. 4). No complications developed in the postoperative period, except transient diabetes insipidus. At 8 months' follow-up after surgery, the patient had no more headache episodes; however, hypogonadism and visual field remain unimproved. Postoperative MRI showed a heterogeneous residual tumor appearance with a remnant of the retroclival dural tail before mentioned (-Fig. 5 ).
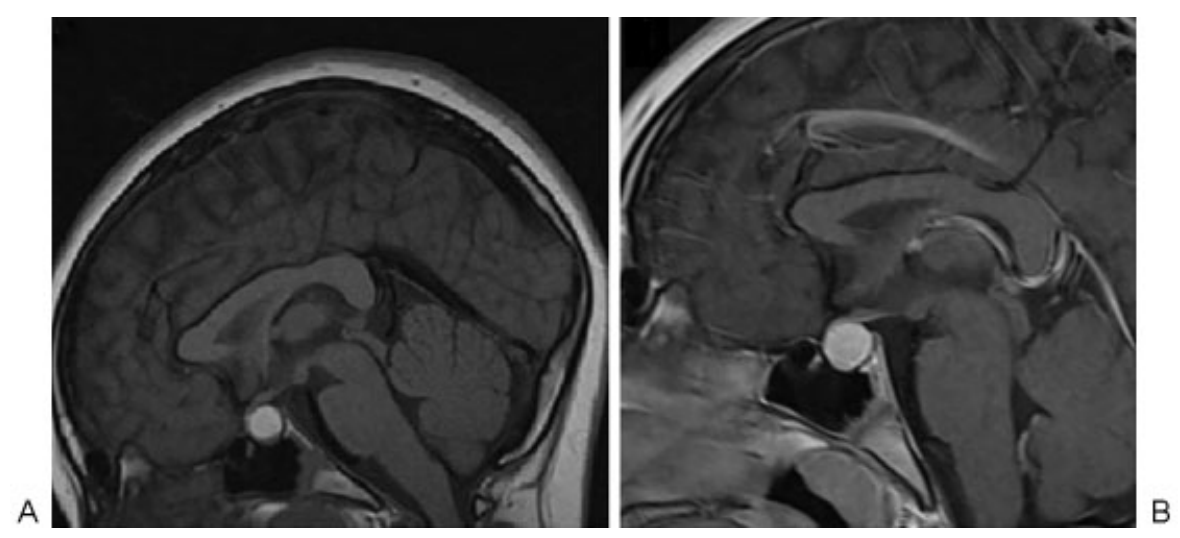

Fig. 2 Case 1. (A) Sagittal T1 precontrast and (B) sagittal T1 postcontrast. Sellar-suprasellar hyperintense cystic tumor with a thin peripheric solid component (A). Solid peripheric component slightly enhanced, cystic component remains hyperintense (B). 
A
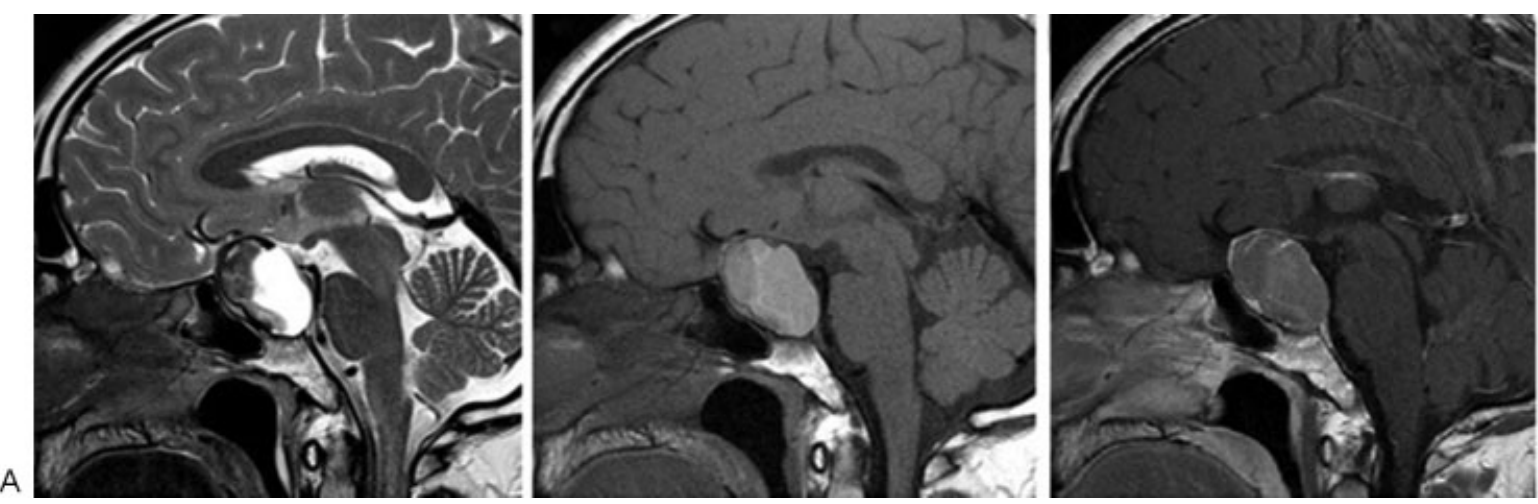

$B, C$

Fig. 3 Case 2. (A) Sagittal T2, (B) sagittal T1 precontrast, and (C) sagittal T1 postcontrast. Sellar-suprasellar solid cystic expansion process with predominance of cystic component hyperintense in T2, and a hypointense peripheric solid component (A). Cystic hyperintense in T1 (B). Solid peripheric component and retroclival dural tail slightly enhanced (C). Mass effect on optic chiasm.

\section{Case 3}

A 31-year-old male patient, without morbid or surgical history, presented with a 2-year history of progressive decrease libido and erectile dysfunction associated with loss of muscle mass and progressive visual loss in the right eye. Endocrinological evaluation showed panhypopituitarism and brain MRI showed a sellar mass contacting the optic chiasm (-Fig. 6).

An extended endonasal endoscopic approach similar to previous cases was performed without complications. Histopathological findings were consistent with xanthogranuloma like the other cases. At 6 months' postoperative followup, the patient persisted with panhypopituitarism and visual deficit without improvement. Postoperative MRI showed complete tumor resection without residual lesion.

\section{Discussion}

The xanthogranulomatous masses in the sellar-parasellar region were considered as a variation of adamantinomatous $\mathrm{CP}^{25}$ Paulus et $\mathrm{al}^{3}$ proposed this pathological pattern as a distinct clinicopathological entity and compared these lesions with CPs. They found several statistically significant differences highlighting the presentation at a younger age, intrasellar location, more severe endocrinological dysfunction, longer preoperative history, lower frequency of visual disturbances, and better surgical resectability with more favorable outcomes. Therefore, this entity was added to the WHO brain tumor classification in 2000 , found mentioned in the third and fourth edition of the WHO classification of tumors of the central nervous system. ${ }^{26,27}$

Most cases of xanthogranulomas of the sellar region have been reported in Asia with few reports in Western Hemisphere and none in Latin America. Therefore, the incidence of xanthogranulomas in the Western Hemisphere remains unknown. There is controversy regarding the origin of these rare lesions, and there are two main hypotheses under greater discussion. The first theory postulates that xanthogranulomas originate from an inflammatory reaction, hemorrhage, or rupture of a RCC. The second theory proposes their origin from a secondary inflammatory progression of a $\mathrm{CP}^{3,13,19,21,24}$ However, there is also reports related to systemic diseases such as sarcoidosis ${ }^{7}$ and Erdheim-Chester's disease. ${ }^{5,16}$ The actual evidence is not yet conclusive regarding its etiology. The presence of squamous epithelium
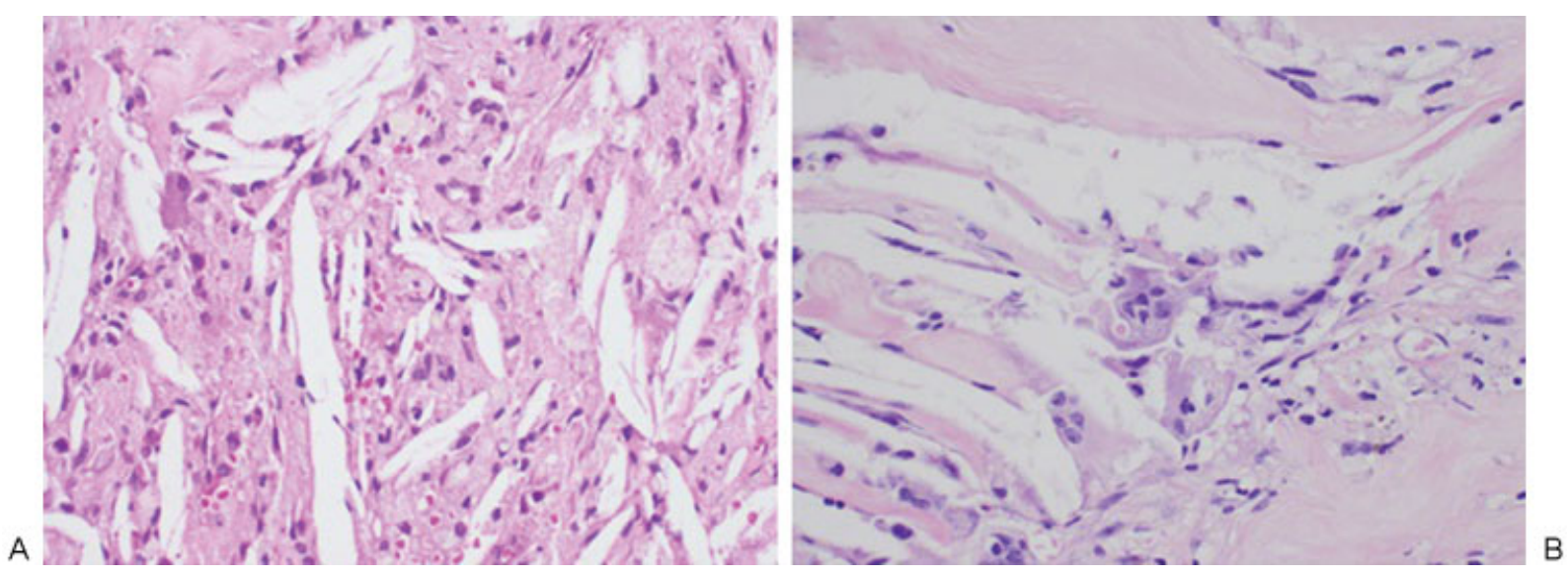

Fig. 4 (A) Hematoxylin-eosin technique, fibrous tissue is observed with xanthogranulomatous chronic inflammation. (B) Crystals of cholesterol, inflammatory cells, and macrophages. 

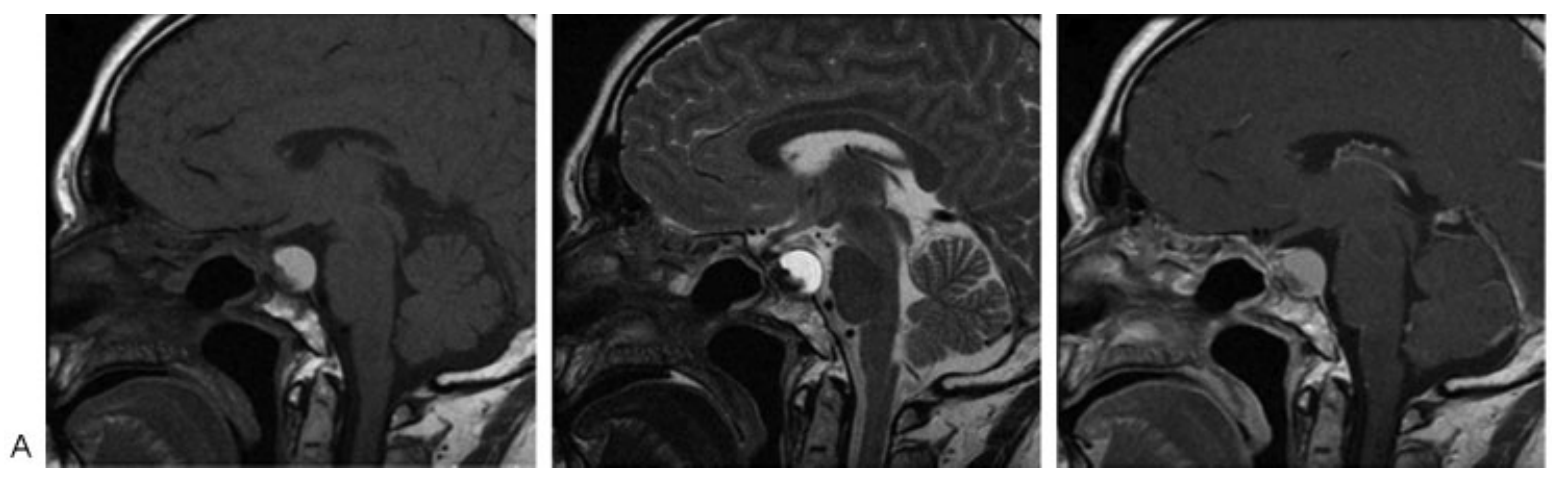

$B, C$

Fig. 5 Case 2. Postoperative (A) sagittal T1 precontrast, (B) sagittal T2, and (C) sagittal T1 postcontrast. Lesion suggestive of residual tumor, smaller than preoperative. Solid cystic sellar expansive process. The anterior thin solid peripheral component shows slightly enhancement postcontrast and remaining retroclival dural tail.

and calcifications reinforce the theory that relate xanthogranulomas to adamantinomatous $\mathrm{CPs},{ }^{3}$ but the evidence gathered last year has been accumulating, favoring their origin related to RCC. ${ }^{1,2}$

Clinically, they are characterized by the presence of cephalea, weight loss, anorexia, nausea, fatigue, visual disturbances, and endocrine disorders ranging from mild deficiencies of one or more hormone to panhypopituitarism. They have also been reported as a cause of diabetes insipidus of central origin (5-10.19) and obstructive hydrocephalus. ${ }^{15}$ All our cases were nonfunctioning tumors. In our series of cases, headache was the predominant symptom in one case, while in the other two cases were secondary to hypogonadism. Due to the rarity of this entity and considering its definitive diagnosis is by biopsy of the surgical specimen, the natural history of presentation of xanthogranuloma remains unknown, and there is no diagnostic method developed to achieve an accurate preoperative diagnosis to date. There are no typical radiological characteristics or patterns for xanthogranuloma. ${ }^{9,20}$ They have variable levels of intensity secondary to unpredictable bleeding patterns and calcified lesions associated; therefore, it has not been possible to describe a typical imaging pattern presented consistently.
We have conducted a literature review of previously reported cases of xanthogranulomas of the sellar-suprasellar region and elaborated a table focusing on the imaging characteristics with the intention of achieving an imaging pattern that contributes to the preoperative diagnosis ( - Table 1), without finding patterns or consistency in its MR signal characteristics. Recently, Madan Mohan et al ${ }^{23}$ reported a case with residual tumor followed up with MR assessment revealing the development of new lesions that may illustrate a growth pattern, one of these lesions described was the development of a dural tail, also developed in our case 2 , supporting it as an interesting feature that may elucidate a new imaging pattern that could be helpful in preoperative diagnosis.

The increased risk of pituitary dysfunction compared with CP and RCC is probably due to bleeding, inflammation, or degeneration of a primary lesion. Regardless of tumor size, it appears that there is a better outcome when there is less intrasellar commitment and time between the onset of symptoms and the surgical resolution. ${ }^{19}$ Surgical resection is the treatment of choice. The management has been almost universally treated by craniotomy or transsphenoidal microscopic surgery. ${ }^{17,19,20}$ Our three cases were treated by endoscopic endonasal transsphenoidal

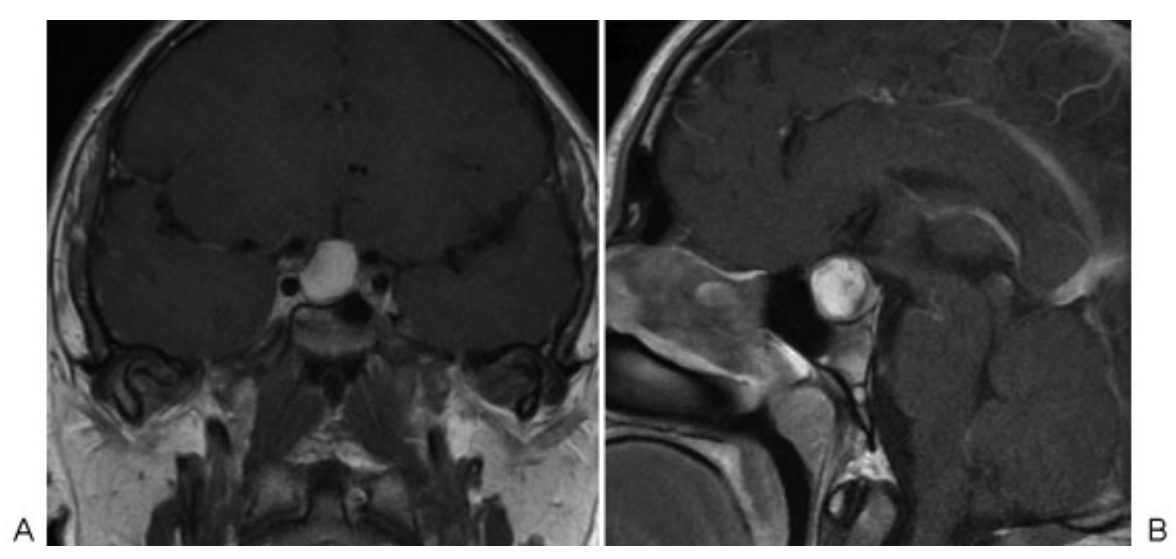

Fig. 6 Case 3. (A) Coronal T1 postcontrast and (B) sagittal T1 postcontrast. Intra- and suprasellar cystic tumor with small solid excentric extension, with predominance of $\mathrm{T} 1$ hyperintense cystic component, and a thin peripheral solid component which slightly enhanced postcontrast. Optic chiasm compression. 


\begin{tabular}{|c|c|c|c|c|c|c|c|c|c|c|c|c|c|c|c|}
\hline 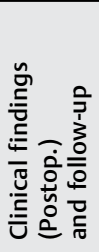 & z & 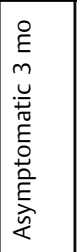 & 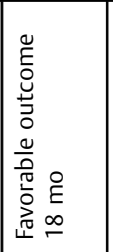 & 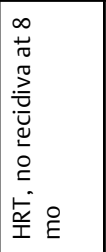 & $\Sigma$ & $\Sigma$ & $\Sigma$ & 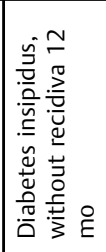 & 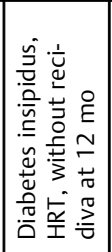 & 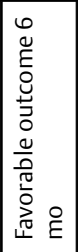 & 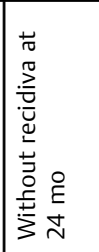 & 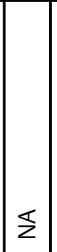 & 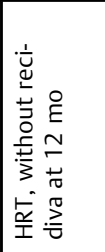 & 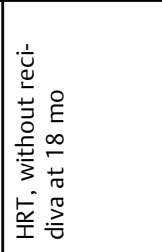 & 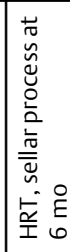 \\
\hline 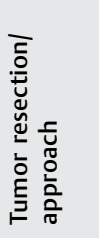 & 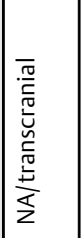 & 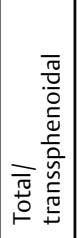 & 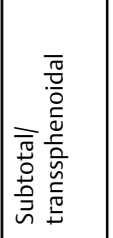 & 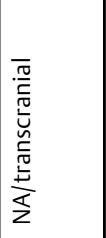 & $\frac{\pi}{z}$ & 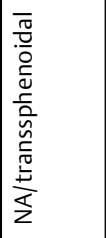 & 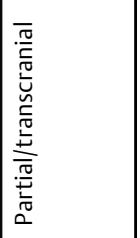 & 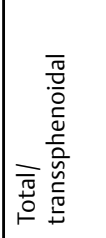 & 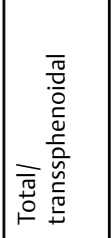 & 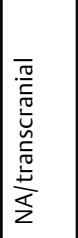 & 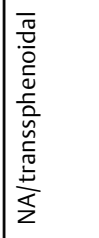 & \& & 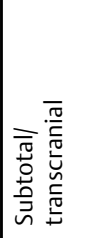 & 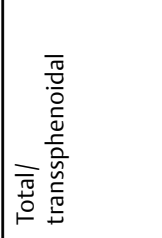 & 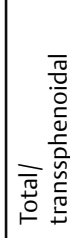 \\
\hline 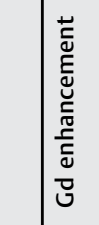 & 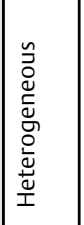 & 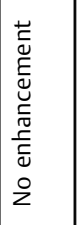 & 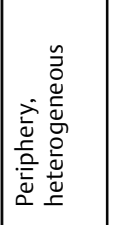 & 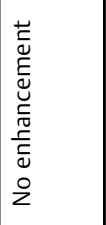 & 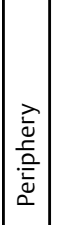 & 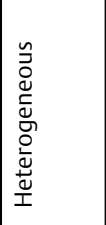 & $\Sigma$ & 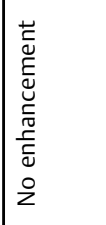 & 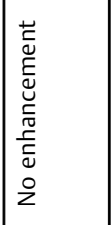 & 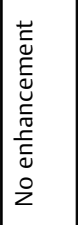 & 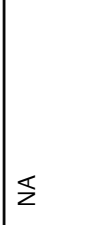 & 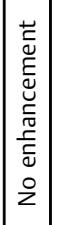 & 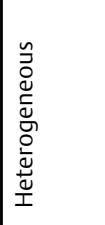 & 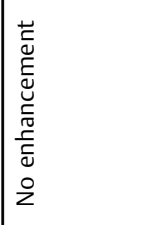 & 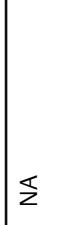 \\
\hline$F$ & $\S$ & 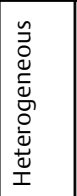 & 乏 & 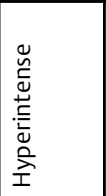 & 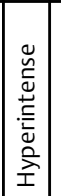 & 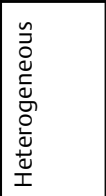 & 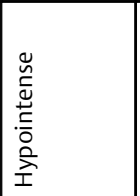 & 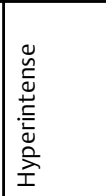 & 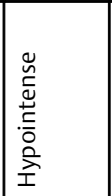 & 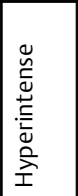 & 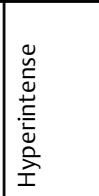 & 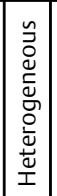 & 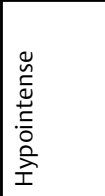 & 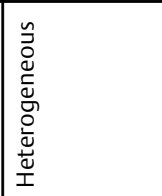 & 文 \\
\hline 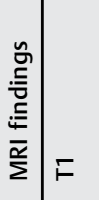 & 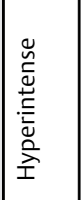 & 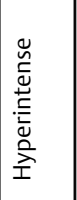 & 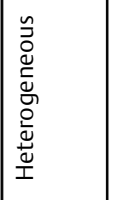 & 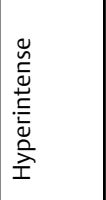 & 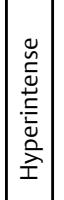 & 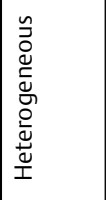 & 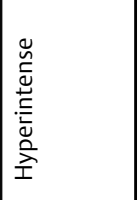 & 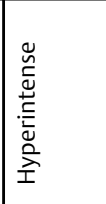 & 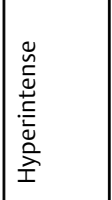 & 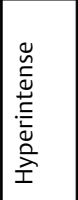 & 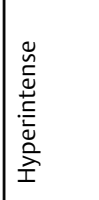 & 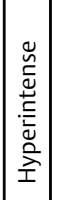 & 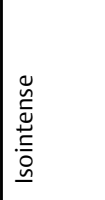 & 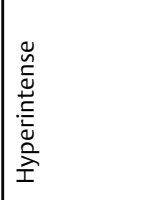 & $\Sigma$ \\
\hline 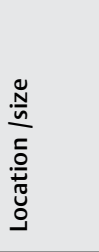 & 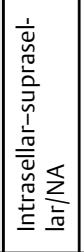 & 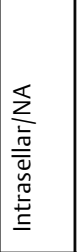 & 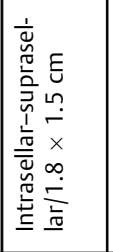 & 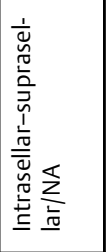 & 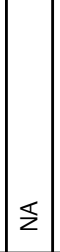 & 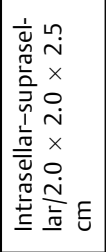 & 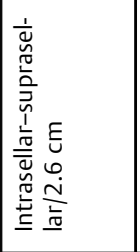 & 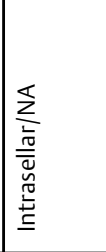 & 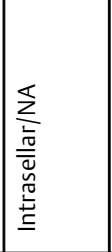 & 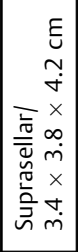 & 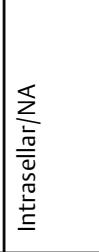 & $\frac{}{z}$ & 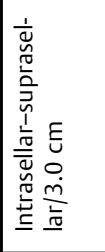 & 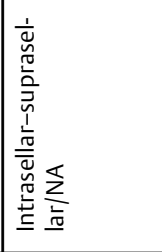 & 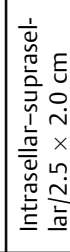 \\
\hline 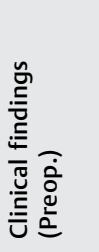 & 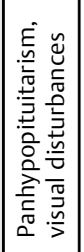 & 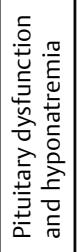 & 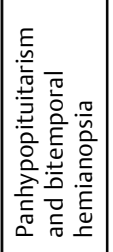 & 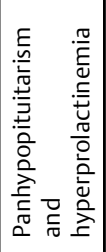 & 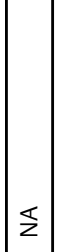 & 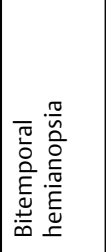 & 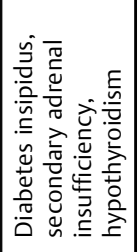 & 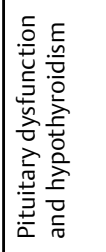 & 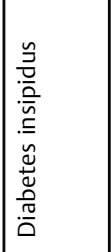 & 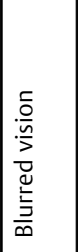 & 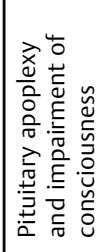 & 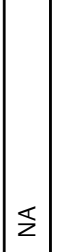 & 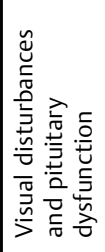 & 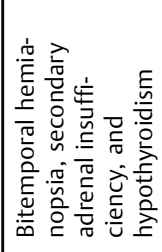 & 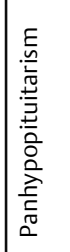 \\
\hline 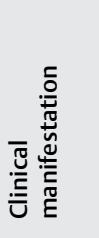 & 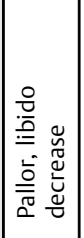 & 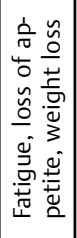 & 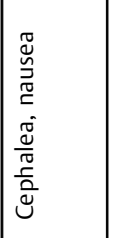 & 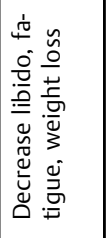 & $\Sigma$ & 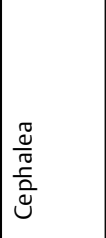 & 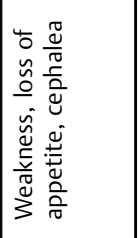 & 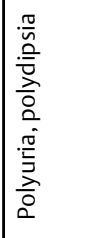 & 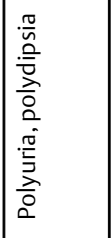 & 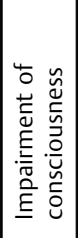 & 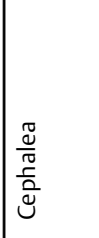 & $\Sigma$ & 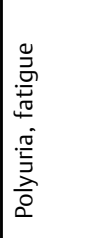 & 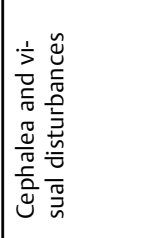 & \\
\hline 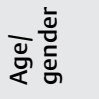 & $\mid \sum \sum$ & $\sum_{\mathbb{6}}$ & $\frac{\sum}{\underset{N}{N}}$ & $\underset{\substack{\infty \\
N}}{\sum}$ & $\mid \underset{\gamma}{\mathcal{F}}$ & $\frac{u}{\sqrt{n}}$ & $\sum_{n}$ & $\sum_{\sigma}$ & $\sum_{0}$ & 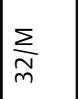 & $\frac{\mathrm{L}}{6}$ & $\underset{⿱ 乛 龰}{\mathrm{f}}$ & $\underset{\substack{0 \\
N}}{\sum}$ & $\frac{\mathrm{U}}{\stackrel{\mathrm{N}}{\mathrm{n}}}$ & $\frac{\sum}{\sigma}$ \\
\hline 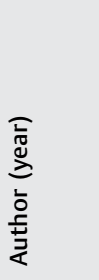 & 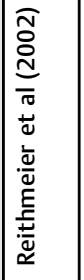 & 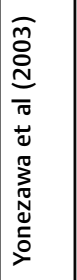 & 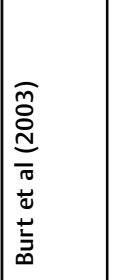 & & 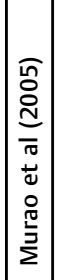 & 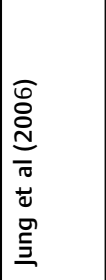 & & 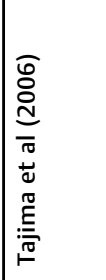 & & 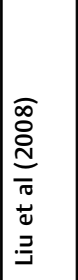 & 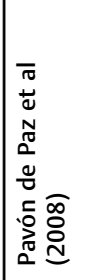 & 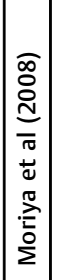 & 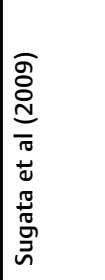 & 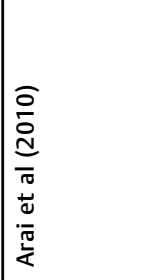 & 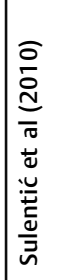 \\
\hline
\end{tabular}




\begin{tabular}{|c|c|c|c|c|c|c|c|c|c|c|c|c|c|c|c|}
\hline 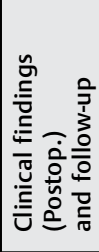 & & $\S$ & 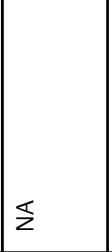 & z & 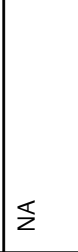 & z & 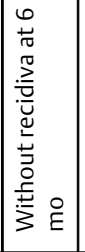 & 产 & 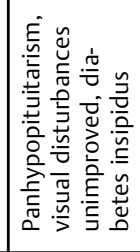 & 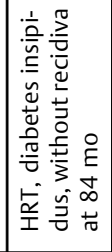 & 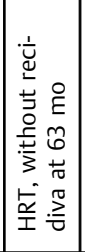 & 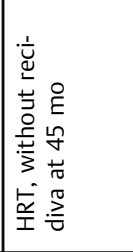 & 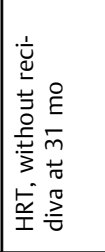 & 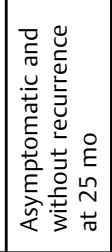 & 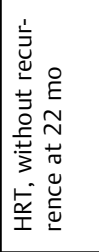 \\
\hline 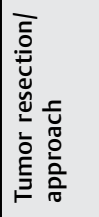 & & 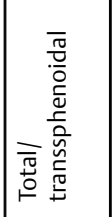 & 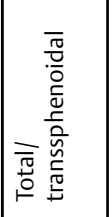 & 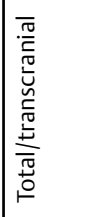 & 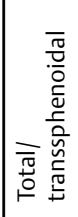 & 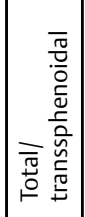 & 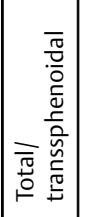 & 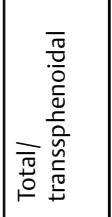 & 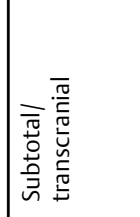 & 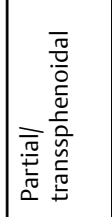 & 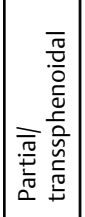 & 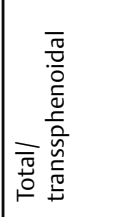 & 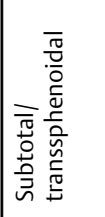 & 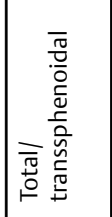 & 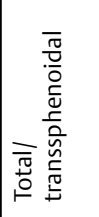 \\
\hline 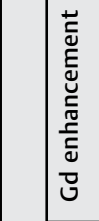 & & 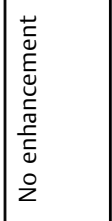 & 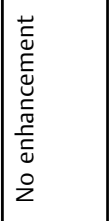 & 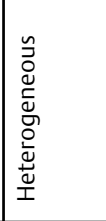 & 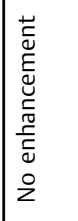 & 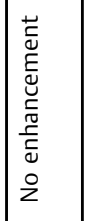 & 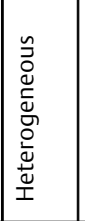 & 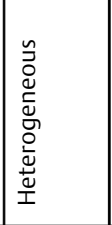 & 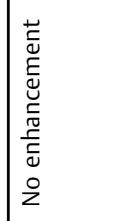 & 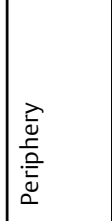 & 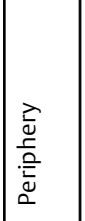 & 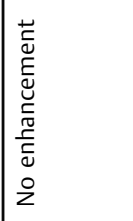 & 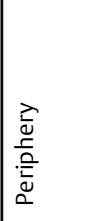 & 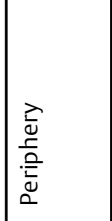 & 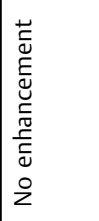 \\
\hline$F$ & & 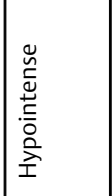 & 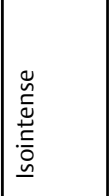 & 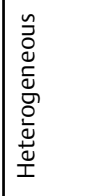 & 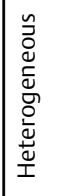 & 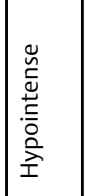 & 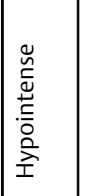 & 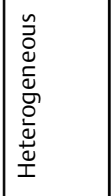 & 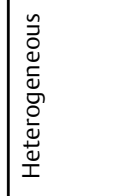 & 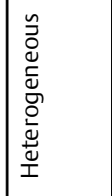 & 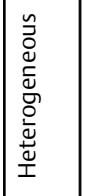 & 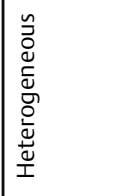 & 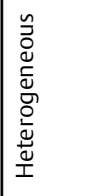 & 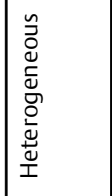 & 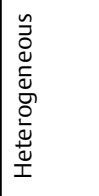 \\
\hline 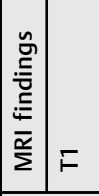 & & 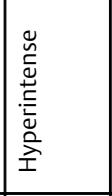 & 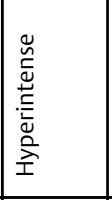 & 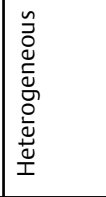 & 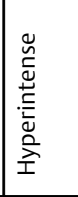 & 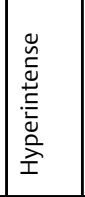 & 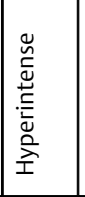 & 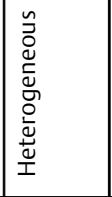 & 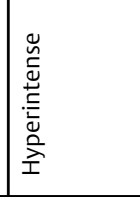 & 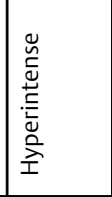 & 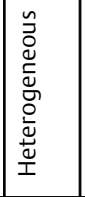 & 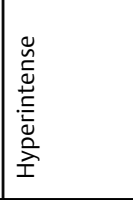 & 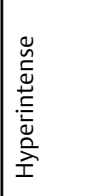 & 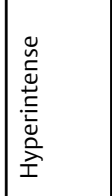 & 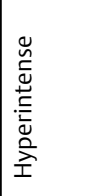 \\
\hline 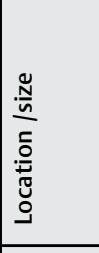 & & 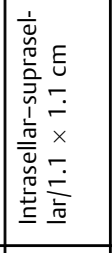 & 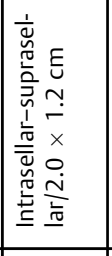 & 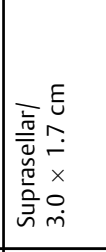 & 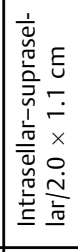 & 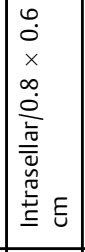 & 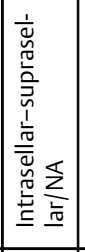 & 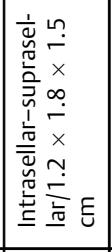 & 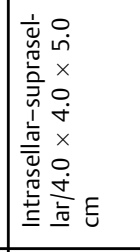 & 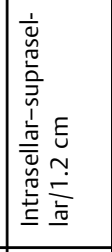 & 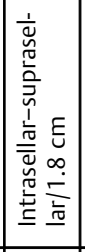 & 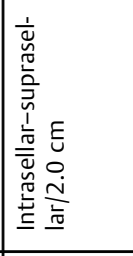 & 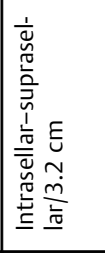 & 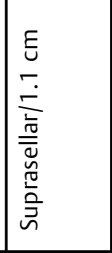 & 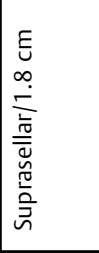 \\
\hline 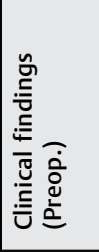 & & 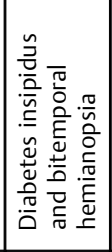 & 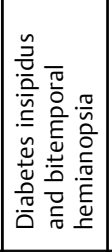 & 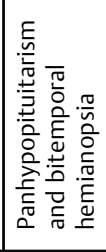 & 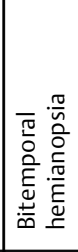 & 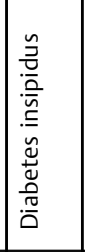 & 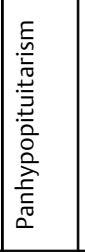 & 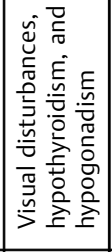 & 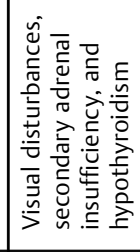 & 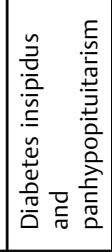 & 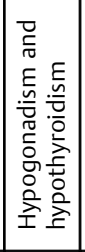 & 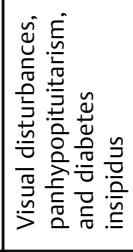 & 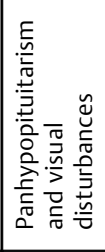 & 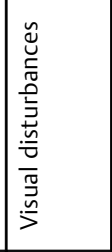 & 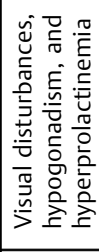 \\
\hline 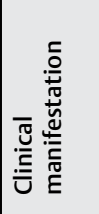 & 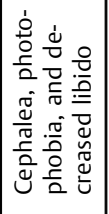 & 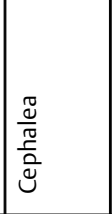 & 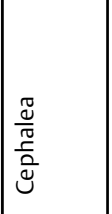 & 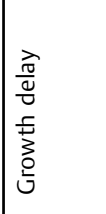 & 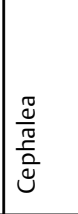 & 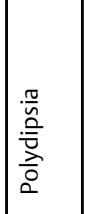 & 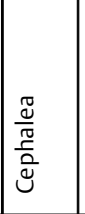 & 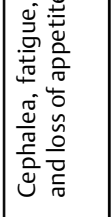 & 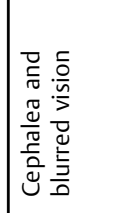 & \begin{tabular}{|l}
$\frac{\pi}{\mathbb{U}}$ \\
$\frac{\pi}{\tilde{U}}$ \\
$\mathbb{U}$
\end{tabular} & 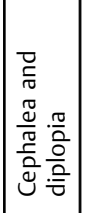 & 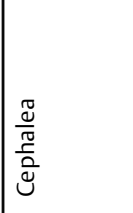 & 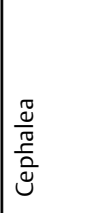 & $\mid \begin{array}{l}\frac{\pi}{\bar{a}} \\
\frac{0}{\underline{O}} \\
\overline{\overline{0}}\end{array}$ & 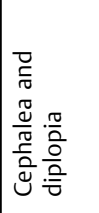 \\
\hline 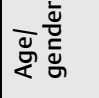 & & $\frac{u}{\infty}$ & $\underset{\Xi}{\Xi}$ & $\stackrel{\stackrel{u}{I}}{=}$ & 殅 & $\sum_{i n}$ & $\sum_{\bar{\sigma}}$ & $\sum_{\mathcal{F}}^{\sum}$ & $\frac{u}{g}$ & 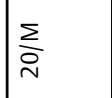 & $\underset{f}{\sum}$ & 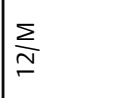 & $\frac{\vec{a}}{9}$ & 㭊 & 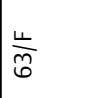 \\
\hline 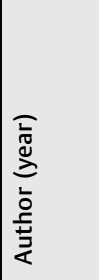 & & 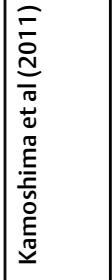 & & & & & 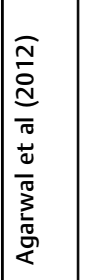 & 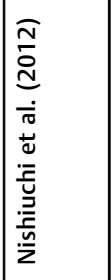 & 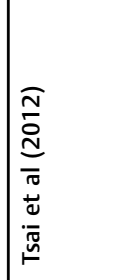 & 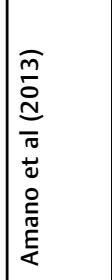 & & & & & \\
\hline
\end{tabular}




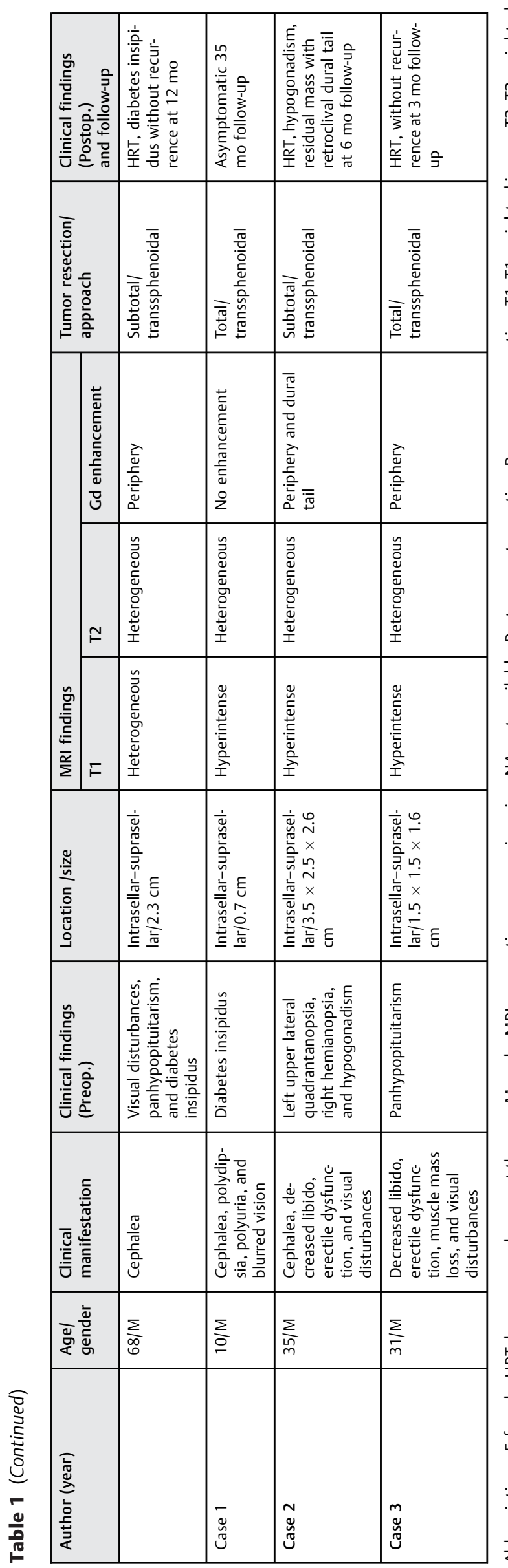

approach achieving a satisfactory exposure with no morbidity related to the surgical procedure, being less invasive than craniotomy. Recurrences after complete resection are rare. ${ }^{16}$ Radiotherapy has also been described as an effective treatment in a case of partial resection. $^{20}$

\section{Conclusion}

Otolaryngologists and neurosurgeons must be cognizant of the existence of sellar xanthogranulomas as a differential diagnosis. We report the first three cases in Latin America, all of them operated by an endonasal endoscopic extended approach which allows a satisfactory exposure and gross tumor resection being a less invasive and effective approach to cure this pathology. The existence of a retroclival dural tail sign in a context of a sellar solid cystic lesions suspicious for a CP or RCC might be a specific feature that could raise the suspicion of a xanthogranuloma. Further studies with MRI assessment are needed to prove the reliability of this imaging characteristic.

\section{Conflict of Interest}

The authors declare no conflict of interest of any kind related to this article.

\section{Funding}

This work was supported financially by University of Concepción, Vice-Rector of Research and Development.

\section{Ethical Approval}

This article does not contain any studies with animals performed by any of the authors. It corresponds to a retrospective study, according to the instructions for the authors for this type of study, formal consent is not required.

\section{References}

1 Le BH, Towfighi J, Kapadia SB, Lopes MB. Comparative immunohistochemical assessment of craniopharyngioma and related lesions. Endocr Pathol 2007;18(01):23-30

2 Rahmani R, Sukumaran M, Donaldson AM, Akselrod O, Lavi E, Schwartz TH. Parasellar xanthogranulomas. J Neurosurg 2015; 122(04):812-817

3 Paulus W, Honegger J, Keyvani K, Fahlbusch R. Xanthogranuloma of the sellar region: a clinicopathological entity different from adamantinomatous craniopharyngioma. Acta Neuropathol 1999; 97(04):377-382

4 Flanagan DE, Ibrahim AE, Ellison DW, Armitage M, Gawne-Cain M, Lees PD. Inflammatory hypophysitis - the spectrum of disease. Acta Neurochir (Wien) 2002;144(01):47-56

5 Reithmeier T, Trost HA, Wolf S, Stölzle A, Feiden W, Lumenta CB. Xanthogranuloma of the Erdheim-Chester type within the sellar region: case report. Clin Neuropathol 2002;21(01):24-28

6 Yonezawa K, Shirataki K, Sakagami Y, Kohmura E. Panhypopituitarism induced by cholesterol granuloma in the sellar region-case report. Neurol Med Chir (Tokyo) 2003;43(05):259-262 
7 Burt MG, Morey AL, Turner JJ, Pell M, Sheehy JP, Ho KK. Xanthomatous pituitary lesions: a report of two cases and review of the literature. Pituitary 2003;6(03):161-168

8 Murao K, Imachi H, Ishida T, et al. [Panhypopituitalism induced by xanthogranuloma of the sellar region]. Nippon Naika Gakkai Zasshi 2005;94(12):2597-2599

9 Jung CS, Schänzer A, Hattingen E, Plate KH, Seifert V. Xanthogranuloma of the sellar region. Acta Neurochir (Wien) 2006;148(04): 473-477

10 Tajima T, Sawamura Y, Ishizu K, Tsubaki J. Two children with xanthogranuloma of the sellar region. Clin Pediatr Endocrinol 2006;15(03):85-91

11 Pavón de Paz I, Peñalver Talavera D, Olivar Roldán J, et al. Sellar xanthogranuloma. Endocrinol Nutr 2008;55(02):102-106. Epub 2008 Oct 14. [Article in English]

12 Moriya S, Nimura T, Utsunomiya A, et al. Xanthogranuloma of the sellar region: a case report and review of literatures. Neurol Surg (Tokyo)18:1024-1028

13 Sugata S, Hirano H, Yatsushiro K, Yunoue S, Nakamura K, Arita K. Xanthogranuloma in the suprasellar region. Neurol Med Chir (Tokyo) 2009;49(03):124-127

14 Arai A, Nishihara M, Sasayama T, et al. Xanthogranuloma of the sellar region-case report. Neurol Med Chir (Tokyo) 2010;50(06): 488-491

15 Liu ZH, Tzaan WC, Wu YY, Chen HC. Sellar xanthogranuloma manifesting as obstructive hydrocephalus. J Clin Neurosci 2008; 15(08):929-933

16 Sulentić P, Cupić H, Cerina V, Vrkljan M. Xanthogranuloma of the sellar region in a patient with sarcoidosis. Acta Clin Croat 2010; 49(01):61-65

17 Kamoshima Y, Sawamura Y, Motegi H, Kubota K, Houkin K. Xanthogranuloma of the sellar region of children: series of five cases and literature review. Neurol Med Chir (Tokyo) 2011;51(10):689-693
18 Agarwal A, Agarwal K, Lee HK. Xanthogranuloma of the sellar region. A case report. Neuroradiol J 2012;25(02):181-184

19 Nishiuchi T, Murao K, Imachi H, et al. Xanthogranuloma of the intrasellar region presenting in pituitary dysfunction: a case report. J Med Case Reports 2012;6:119

20 Tsai TS, Tsui YK, Wu MWL, et al. Xanthogrnauloma of sellar region: a case report with diagnostic imaging findings, treatment response and literature review. J Radiol Sci 2012; 37:89-94

21 Amano K, Kubo O, Komori T, et al. Clinicopathological features of sellar region xanthogranuloma: correlation with Rathke's cleft cyst. Brain Tumor Pathol 2013;30(04):233-241

22 Miyajima Y, Oka H, Utsuki S, Fujii K. Rathke's cleft cyst with xanthogranulomatous change-case report. Neurol Med Chir (Tokyo) 2011;51(10):740-742

23 Madan Mohan B, Mohamed E, Jain SK, Jain M, Jaiswal AK. Serial MR imaging in suprasellar xanthogranuloma: growth pattern and new lesions. J Neuroimaging 2015;25(04):677-679. doi: 10.1111/ jon. 12170

24 Burger PC, Scheithaeur BW, Vogel FS. Surgical Pathology of the Nervous System and Its Coverings, 3rd ed. New York: Churchill Livingstone; 1991:537-545

25 Al-Khalidi H, Hawkins C. Histopathological features of common pediatric brain tumors. In: Mehta MP, ed. Principles \& Practice of Neuro-Oncology: A Multidisciplinary Approach. New York: Demos Medical Publishing; 2010:204-214

26 Rushing EJ, Giangaspero F, Paulus W, et al. Craniopharyngioma. In: Louis DN, Ohgaki H, Wiestler OD, Cavenee WK, eds. WHO Classification of Tumors of the Central Nervous System, 4th ed. Lyon, France: IARC Press; 2007:238-240

27 Kleihues P, Cavenee WK, eds. Pathology and genetics of tumours of the nervous system. World Health Organization Classification of Tumours. Lyon, France: IARC Press; 2000 